\title{
DECIMETER LEVEL MAPPING USING DIFFERENTIAL PHASE MEASUREMENTS OF GPS HANDHELD RECEIVERS
}

\author{
Dr. Ahmed El-Mowafy \\ Civil and Environmental Engineering Department \\ College of Engineering \\ The United Arab Emirates University \\ Ahmed.Mowafy@uaeu.ac.ae
}

\begin{abstract}
An economic positioning system is presented in this study for decimeter level accuracy mapping with emphasis on producing base maps for GIS applications. The system downloads carrier-phase data of GPS handheld receivers online, simultaneously registers the point code and description, and processes the data in a differential post-mission mode. The architecture of the system is presented. Characteristics of the proposed system are discussed. Main issues in system utilization are investigated, including: treatment of system errors, phase measurements ambiguity resolution, the impact of receiver dynamics on system performance, and the required occupation time per point. Some guidelines for system operation are proposed. Several tests in the static and kinematic modes were carried out to test the performance of the proposed system. The system gave very promising results. Test results show that for a probability of 95\%, an accuracy of less than $16 \mathrm{~cm}$ can be achieved after correctly resolving the ambiguities. With a cost that is a fraction of that of the currently used geodetic-grade GPS receivers, the system represents a cheap alternative for a wide range of GIS applications.
\end{abstract}

\section{INTRODUCTION}

Mapping by GPS is usually carried out using geodetic-grade receivers. One important application is producing maps and registering the data spatially in GIS applications. [12] and [1] demonstrated that positioning by GPS can achieve a good balance of accuracy, currency, and completeness against cost and level of effort in GIS spatial data registration. Fieldwork cost can further be decreased if the widely-used geodetic receivers are replaced by cheap handheld receivers. These receivers are mainly used for low-accuracy navigation in a single positioning mode using code measurements. However, they are not suitable for a wide range of GIS applications because of their low accuracy. This accuracy can reach the sub-meter or the decimeter level by downloading the code and phase measurements of handheld receivers and processing them, coupled with measurements from a reference receiver, in a differential post-mission scheme.

A system was built to perform this task. A unit of the proposed system consisting of an off-the-shelf handheld receiver, a downloading cable, a low-cost notebook or a palmtop computer, and a downloading software license would cost approximately US $\$ 1500$ or even less. In addition, the system has little weight and size. The system is suitable for updating maps, particularly when gathering dense geographic data in open areas. In addition to GIS, the system can be used for medium accuracy mapping, or as a backup system to other mapping techniques. It will also help in spreading the use of GPS in positioning in poor countries or small firms, where an investment in a relatively expensive geodetic-grade GPS system might be difficult.

\section{DECIMETER POSITIONING BY DIFFERENTIAL PHASE DATA FROM GPS-HANDHELD SYSTEMS}

Handheld receivers are mainly designed for navigation applications, and hence they usually employ single frequency code measurements for positioning. They can also be augmented with Doppler or carrier phase measurements for improving velocity 
determination and for the smoothing of results. Positioning accuracy with handheld receivers in a typical point positioning approach can thus be in the range of a few meters. However, to utilize handheld GPS receivers for mapping and GIS applications, measurement accuracy at the $\mathrm{cm}$ to decimeter level is needed. The use of GPS measurements in a differential mode thus becomes mandatory.

A system was built with such a capability, comprising a handheld receiver, a downloading cable, and a notebook (or a Palmtop) for storing data. Two types of software were simultaneously used in the field, one for recording the GPS code and phase measurements online, and the second for recording the occupied points code and information. The collected data is post-processed in a differential mode combined with data from another handheld or geodetic-grade receiver occupying a reference station. Thus, most of the errors that contribute to lowering the system accuracy are reduced. The positioning accuracy can be accordingly improved to the sub-meter level using code measurements, and to the decimeter level using phase measurements. This accuracy is adequate for the majority of GIS applications. However, with focus on decimeter level accuracy in GIS mapping, the emphasis in this context is given to the collecting and processing of carrier phase data.

\section{Real-time Data Logging in Handheld Receivers}

Downloading code and phase measurements is an essential key to using handheld receivers in a differential mode. To complete this task, a commercial software utility known as GRINGO (GPS RINEX Generator) [6], developed at the IESSG Institute, the University of Nottingham is used. The software downloads the pseudorange and carrier phase data of Garmin 12 channels handheld receivers online in a standard RINEX format using the Garmin communications protocol. Online downloading is necessary since handheld receivers do not store data in an internal memory. [7] reported that by post-processing the Garmin-receivers carrier phase measurements, coordinate accuracy of $10 \mathrm{~cm}$ or better can be achieved. They also demonstrated that for short baselines, the carrier phase float solution, after gathering sufficient phase data in a static mode, can give baselines accurate to the millimeter level. They also argued that the same atmospheric errors had affected equally the handheld receivers (Garmin 12XL) and an 'Ashtech' geodetic-grade receiver.

Another software utility is simultaneously used with the downloading software for entering and storing the occupied point information (its description and code). Point description includes its identifier and special conditions, and point code is used to describe its type, group, symbol, and possibly its attributes. To link the measurements to point details, the GPS downloading software synchronizes the computer clock to the GPS time frame, and at the same time, the point registry software stores the computer time while entering point information. Thus, the positions computed from GPS measurements are linked to their corresponding information.

\section{System Architecture}

Figure 1 illustrates the proposed GPS-handheld positioning system, which consists of:

- A Garmin handheld receiver capable of receiving measurements and data from 12 satellites simultaneously. Three Garmin-receiver models were tested for this purpose (Garmin 12, Garmin 48, and Garmin 76S). The first two models proved to have compatible characteristics, while Garmin $76 \mathrm{~S}$ has a better performance in locking to satellites. The handheld receiver is fixed on a GPS swivel base, mounted on a camera tripod. The receiver is plumbed on the occupied point using a plumb bob or a simple laser pointer.

- A GPS-to-computer downloading cable. 
- A low-cost notebook or a Palmtop computer for point coding and online downloading of GPS measurements.

- The downloading software (GRINGO), version 2.0.0.

- An offset bar with a pre-defined length $(85 \mathrm{~cm})$. This bar is attached to the GPS receiver and is used to offset GPS computed positions to the required points when mapping high buildings to allow for better satellite visibility.

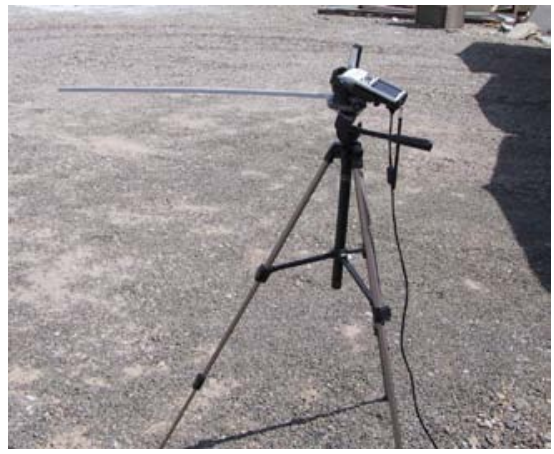

Fig. 1 The GPS handheld positioning system

The system uses only single frequency measurements with an output rate limited to $1 \mathrm{~Hz}$. Studying the output phase measurements shows that they have more noise than phase measurements of the geodetic-grade receivers. In addition, testing the system shows that the satellite signal locking by the Garmin 12 channel receivers is sensitive to dynamics of motion. This means that in the static mode, when no dynamics is experienced, these receivers can maintain constant lock on all visible satellites, until a change of geometry takes place. On the other hand, during motion, some satellites, particularly those of low signal-to-noise ratio and low elevation angles, can frequently experience loss of lock, and thus the number of observed satellites repeatedly varies. This has two implications. First, re-initialization of carrier phase ambiguities and cycle slip correction become frequent. Second, lowering the number of observed satellites has an inverse impact on positioning accuracy, noting that in cases when the number of satellites observed is less than 4, position determination cannot be performed.

\section{TREATMENT OF SYSTEM ERRORS AND AMBIGUITY RESOLUTION}

\section{Error Treatment}

Due to the fact that the proposed GPS-handheld positioning system uses only single frequency phase measurements of relatively high noise, efforts should be directed towards minimizing the impact of traditional errors to maximize achievable accuracy and aid ambiguity resolution. Of particular interest are the spatially correlated errors, e.g. the orbital, tropospheric and ionospheric errors. A simple approach in this regard is to reducing the baseline length, i.e. to use a reference station close to the survey area. If no nearby reference stations are available, a temporary one can be established. Its position can be determined later in the office, and referenced to a known station, which could be a few kilometers away.

Alternatively, since processing is carried out in a post-mission mode, precise orbits can be used to eliminate orbital errors, such as IGS and NGS delayed or rapid orbits, which usually have an RMS of a few centimeters. On the other hand, the impact of tropospheric and ionospheric errors can be minimized by modeling. The troposphere delay being modeled in the zenith direction consists of a hydrostatic component that is responsible for about $90 \%$ of the total delay, and a wet component. The latter 
component is highly variable and it is almost impossible to model it solely from surface measurements [8]. The former tropospheric component can be computed by measuring meteorological data at the antenna site (mainly pressure, temperature and relative humidity), in addition to the latitude and height of the station. Estimation of this tropospheric error can be made by different approaches with varying levels of complexity, see for instance [11]. Numerical weather models present another alternative, where instead of applying default meteorological values taken from the standard atmosphere, latitude-dependent mean values are employed including modeling of seasonal variations. The main advantage of such an approach is that no measurements are needed, which is practical for a lot of applications and cost-effective [9].

The ionospheric error, when using hand-held receivers, cannot be reduced by encountering a linear combination of L1 and L2 phase measurements as usually applied by dual frequency geodetic-grade GPS systems. The standard ionospheric model, which employs parameters transmitted with the GPS message, is also unlikely to eliminate more than $75 \%$ of the ionospheric error, especially during high ionospheric activity periods. Therefore, more efficient models are required.

In this respect, corrections estimated in post mission from data of real-time networks can be utilized. In this method, the archived corrections for code and phase observations estimated at the network reference stations can be downloaded by the rover from a service provider and be used during post processing of field measurements. The rover observations can then be corrected taking into consideration its position and local time. This can be considered as an inexpensive option, bearing in mind the expanding implementation of real-time networks, and a good coverage of these networks in most major cities currently exists or is expected in the near future. There are different methods for computation of the corrections at the user location, either computing a total measurement correction, or a separate value for each error source. Among these methods are: the virtual reference system (VRS), the area parameterization method (FKP), the correction grid method, and the correction function method. For more details of these methods, the interested reader may refer to [5], [3], [10], [2], and [4]. These methods can be simplified in post processing for the case in hand taking into consideration the knowledge of the precise orbits and elimination of the need for interpolation of data. In addition, no data latency is experienced, the data can be filtered recursively, and smoothing can be applied with a larger amount of data.

\section{Ambiguity Resolution}

The feasibility of positioning using phase measurements depends to a great extent on the successful resolution of carrier phase ambiguities. The method adopted in this research is the Least-squares Ambiguity De-correlation Adjustment method (LAMBDA). The approach taken with this method is to re-parameterize the integer least-squares problem. An ambiguity transformation is constructed that tries to decorrelate the original ambiguities to simpler ambiguity elements. The solution is then carried out for the de-correlated ambiguities, resulting in a faster and easier, yet accurate, process [14]. However, when solving the integer ambiguities using the handheld phase measurements, one should consider that only the L1 single frequency measurements are used and they have relatively high noise. Thus, both the volume of the ambiguity search space and values of the ambiguity covariance matrix used have to be greater than when using traditional phase data. It would be hard then to fix an "integer" number of cycles; instead a float solution scheme will be more realistic. 
As a result of the increased search volume, the number of candidate ambiguities will increase, and more data will be needed. Processing in post mission is therefore more feasible due to its advantages. For instance, as much data as needed can be used for ambiguity initialization, and the solution for their respective epochs can be obtained recursively after solving for the ambiguities. Processing time will also not be of concern. In addition, the search region for the correct ambiguities can be minimized by employing precise orbits, which usually give a more accurate initial position.

To assist in finding the correct ambiguity set at selected locations along the surveying route, a constraint can be established by measuring the distance between two consecutive points. The computed positions from any candidate ambiguity set should then satisfy their pre-measured distance within an acceptable tolerance (e.g. $10 \mathrm{~cm}$ ). This constraint can be formulated as:

$$
b_{i-j}=\left(\Delta X_{i-j}^{2}+\Delta Y_{i-j}^{2}+\Delta Z_{i-j}^{2}\right)^{1 / 2}+\delta
$$

where: $b_{i-j}$ is the known baseline length between the positions $\mathrm{i}$ and $\mathrm{j}$, and $\left(\Delta X_{i-j}, \Delta Y_{i-j}\right.$, $\left.\Delta Z_{i-j}\right)$ are their baseline vector components in the WGS-84 frame, based on the tested candidate ambiguity sets, and $\delta$ denotes the pre-set tolerance. In addition, utilization of a simple measure of ambiguity strength, such as the 'Ambiguity Dilution of Precision' (AmDOP) can be helpful in the process of ambiguity validation testing along with traditional tests. This can be performed by comparing the AmDop value for different candidate ambiguity sets and selecting the sets of best AmDop values. In the single frequency case, the AmDOP can be computed in cycles as [13]:

$$
\mathrm{AmDOP}=\mathrm{m}^{\frac{1}{2(\mathrm{~m}-1)}} \sqrt{\frac{\sigma_{\phi 1}^{2}}{\mathrm{~K} \lambda_{1}{ }^{2}}}\left(1+\frac{1}{\varepsilon_{1}}\right)^{1 / 2}
$$

where:

$\begin{array}{ll}\mathrm{m} & \text { the number of satellite tracked } \\ \mathrm{k} & \text { the number of observation epochs } \\ \sigma_{\phi 1}^{2} & \text { the standard deviation of the single phase observables on L1 } \\ \lambda_{1} & \text { the L1 signal wavelength } \\ \varepsilon_{1} & \text { the phase-code variance ratio which equals }\left(\sigma_{{ }_{1} 1}^{2} / \sigma^{2}{ }_{p 1}\right)\end{array}$

\section{TESTING THE PROPOSED SYSTEM}

Testing the performance of the proposed system for medium-accuracy mapping and generation of GIS base maps was carried out for an area surrounding the College of Engineering, Al Jimi1 Campus, the United Arab Emirates (UAE) University. The system performance was investigated in both the static and kinematic modes.

\section{Static Testing}

The handheld positioning system was initially tested in a static mode for approximately 52 minutes. The reference receiver used was an Ashtech dual-frequency receiver set up at a point of pre-determined coordinates located on the roof of the Civil Engineering Department building at the UAE University. The handheld receiver used was a Garmin 48. It occupied a point in an open parking area with $32 \mathrm{~m}$ separation from the reference point to minimize the spatially correlated errors. Figure 2 illustrates 
the satellite scan map of the received satellite data (locking to satellites with time) using the rover handheld receiver. The figure shows that the receiver had a good lock on the observed satellites, and no major cycle slips were seen in the static mode. This fact is illustrated in the continuity of the raw data (code-carrier phase) measurements of the tracked satellites, which are depicted for the satellites numbers 4 and 27, given as examples, in the Figures 3 and 4, respectively.

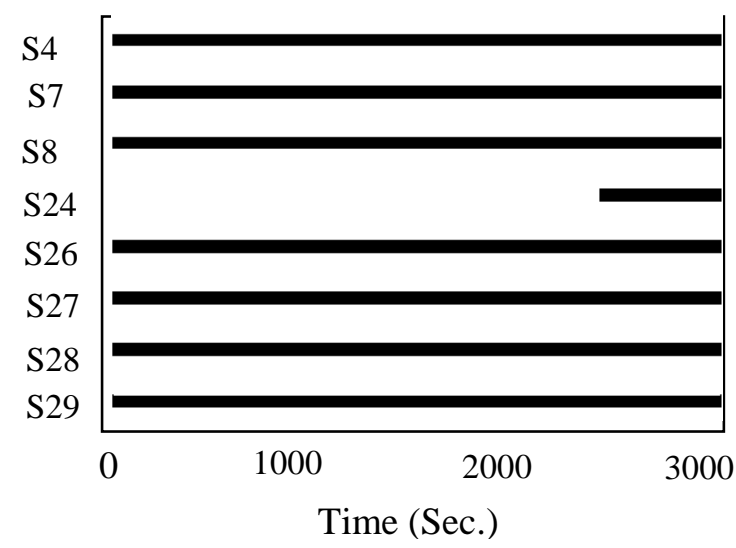

Fig. 2 Satellite scan (lock) for the static test

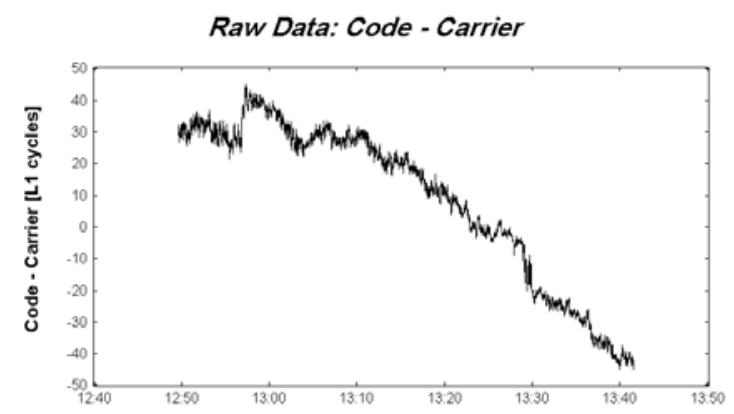

Fig. 3 Changes in raw data for satellite No. 4 (ref.)

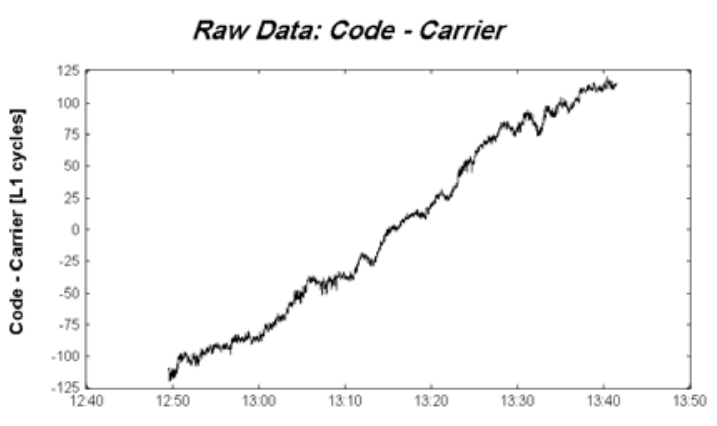

Fig. 4 Changes in raw data for satellite No. 27

The collected data of the reference and the handheld receivers were next post processed in the office. An On-The-Fly (OTF) approach was used for phasemeasurements ambiguity resolution, similar to kinematic processing, where the point coordinates were estimated for each epoch. Table 1 gives the average values of standard deviations of the computed Cartesian position components in the WGS-84 frame $(\mathrm{x}, \mathrm{y}, \mathrm{z})$ and the resulting positions of the handheld receiver as computed from the estimation process. In addition, the table gives the maximum standard deviations computed within the whole data processing. Results show that the RMS of the computed position components was generally less than $5 \mathrm{~cm}$. This precision is less than that of the geodetic-grade receivers, which usually lies in the range of a few millimeters to $2 \mathrm{~cm}$. Noting that the spatially correlated errors were practically eliminated in measurement differencing, the low positioning precision can be attributed to a high noise level in the collected phase measurements of the hand-held receivers (including the measurement random errors, multipath, imaging, and antenna phase-centre variation). This can be seen in the Figures 5 and 6, which show in one static test, for satellite PRN28 as an example, the epoch-to-epoch difference in code and carrier phase measurements from a geodetic-grade receiver and a Garmin 48 handheld receiver, respectively. As Figure 5 shows, these differences ranged between $+/-0.10$ cycle for the geodetic-grade receiver, while they were generally between +/2.5 cycles with high noise for the handheld receiver case, as depicted in Figure 6. 


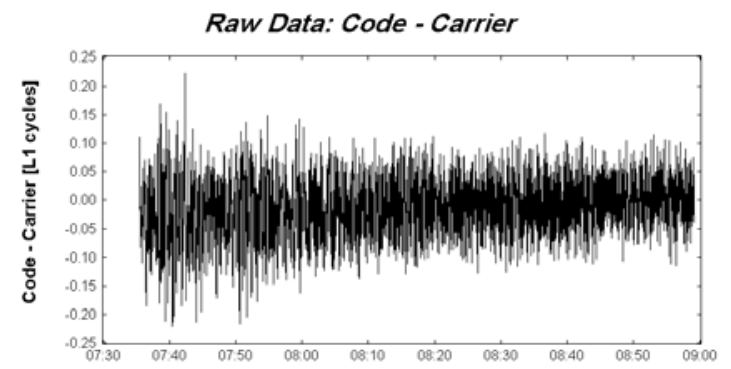

Fig. 5 Phase data changes using a geodetic-grade receiver

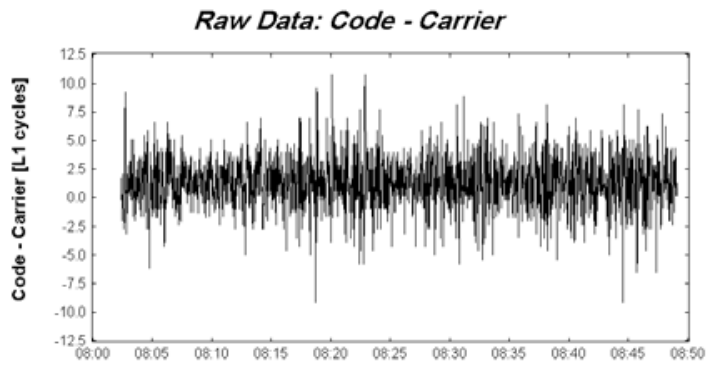

Fig. 6 Phase data changes using a handheld receiver

Table 1. Statistics of positioning precision of the proposed system in the static mode

\begin{tabular}{cccc|cccc}
\hline \hline \multicolumn{3}{c|}{ Average St. Dev. (m) } & \multicolumn{4}{c}{ Maximum St. Dev. (m) } \\
\hline$\sigma_{\mathrm{x}}$ & $\sigma_{\mathrm{y}}$ & $\sigma_{\mathrm{z}}$ & $\sigma_{\mathrm{P}}$ & $\sigma_{\mathrm{x}}$ & $\sigma_{\mathrm{y}}$ & $\sigma_{\mathrm{z}}$ & $\sigma_{\mathrm{P}}$ \\
0.013 & 0.032 & 0.030 & 0.046 & 0.018 & 0.061 & 0.048 & 0.077 \\
\hline
\end{tabular}

\section{Kinematic Testing}

In kinematic testing of the proposed system, the handheld receiver occupied each point for a few seconds while kept locked on the satellite during motion, and cycle slip detection and repair of phase measurements were continuously carried out. Initial ambiguity resolution was executed on-the-fly and re-initialization was performed whenever the number of observed satellites changed. This mode of observation is of main concern since mapping using GPS is usually performed in this mode to achieve a high productivity level. Multiple tests were thus carried out taking the same test location of the static testing. The mapped area is shown in Figure 7. During testing, the proposed system determined positions of pre-marked points that represent the details of the mapping area. The positions of these points were previously determined by an RTK survey using geodetic-grade GPS receivers with 1-3 cm accuracy. To investigate the handheld system performance, its results were compared to the RTK positions, taking the latter as the reference for comparison. Different occupation times per point were also tested. The results of four tests are introduced in this context to summarize the findings. These tests were carried out on October 10, 23 and 30, and on November 6, 2002.

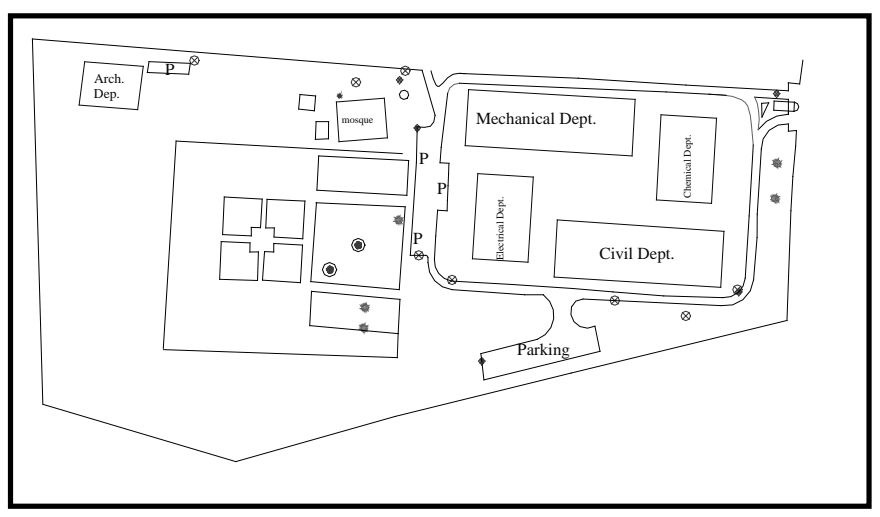

Fig. 7 Kinematic test area 
Figures 8 and 9 show the satellite scan and number of satellites observed during the October 10 test. In this test, point occupation time was limited to five seconds or less. As can be seen from the figures, the number of tracked satellites varied significantly when the handheld receiver experienced dynamics (velocity and acceleration changes, and vibrations). This is clearly illustrated in Figure 8 where the loss and gain of signals was frequent, particularly for low-elevation satellites. This is also depicted in the Figures 10 and 11, which give the measured raw data of satellites number 1 and 8 , respectively. These satellites are two examples of satellites suffering from frequent data discontinuity and cycle slips. As a result, frequent re-initialization of their phase measurement ambiguities were needed, which required several seconds of observations on-the-fly to be correctly solved for each case. In addition, the reduction of the number of observed satellite measurements had a direct impact on reducing the positioning accuracy achieved. As shown in Figure 12, for the first 1148 epoch, when point positions were estimated based on observing 8 to 10 satellites, the $3 \mathrm{D}$ positioning errors were limited to less than $6 \mathrm{~cm}$. On the other hand, after the epoch 1148, the number of satellites observed was generally less than four, and positioning was thus unsuccessful, which resulted in the spikes seen in the figure. The Oct. 23 test gave similar results, where the same occupation time per point was adopted ( $<5$ seconds).

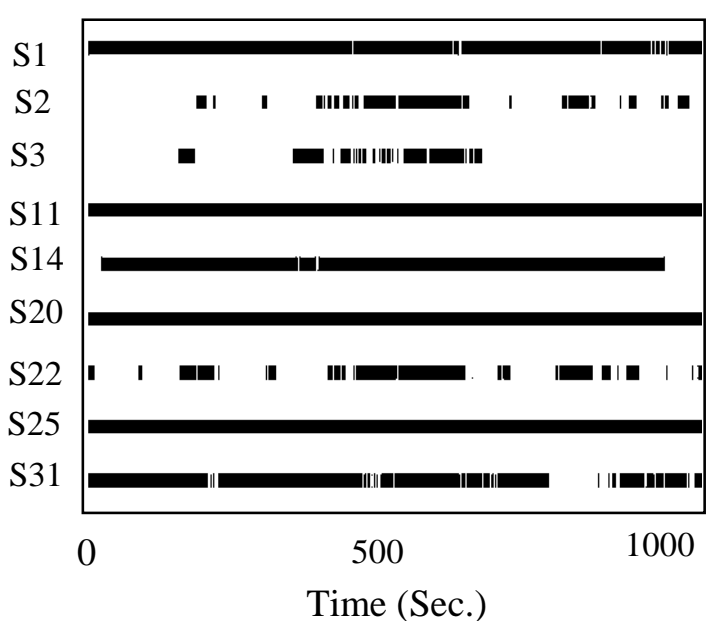

Fig. 8 Satellite scan for Oct. 10 test

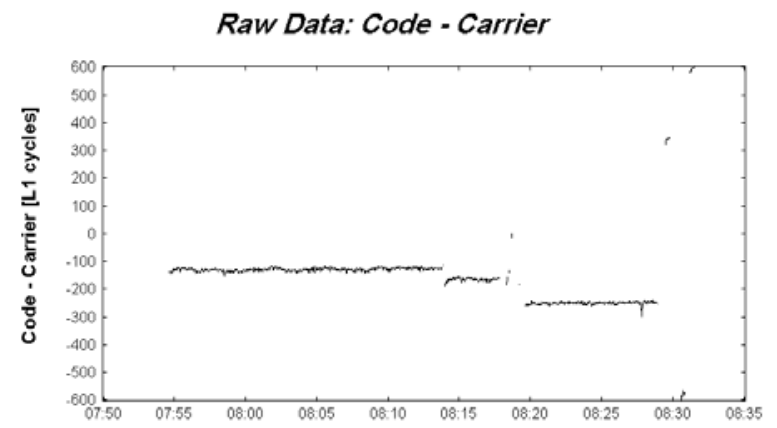

Fig. 10 Changes in raw data for Satellite No. 1

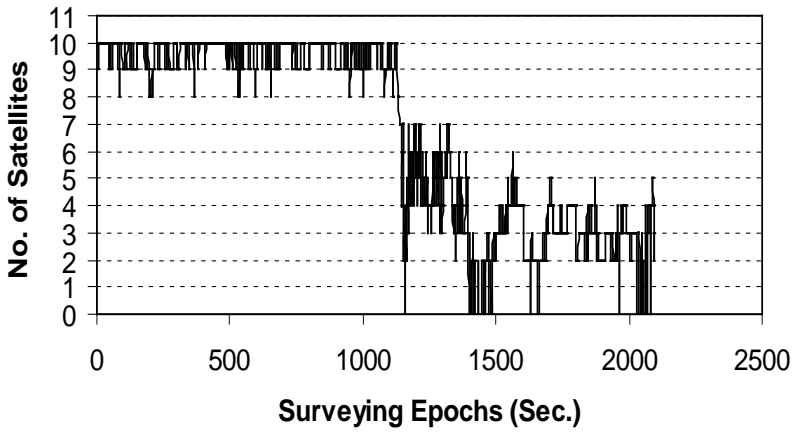

Fig. 9 No. of observed satellites in Oct. 10 test

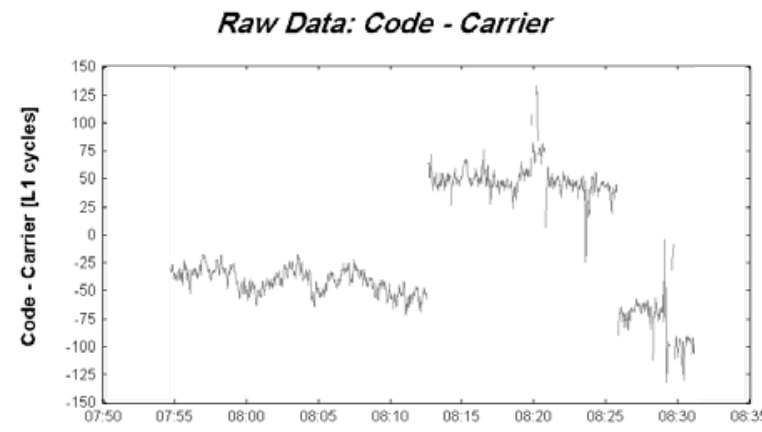

Fig. 11 Changes in raw data for Satellite No. 8 


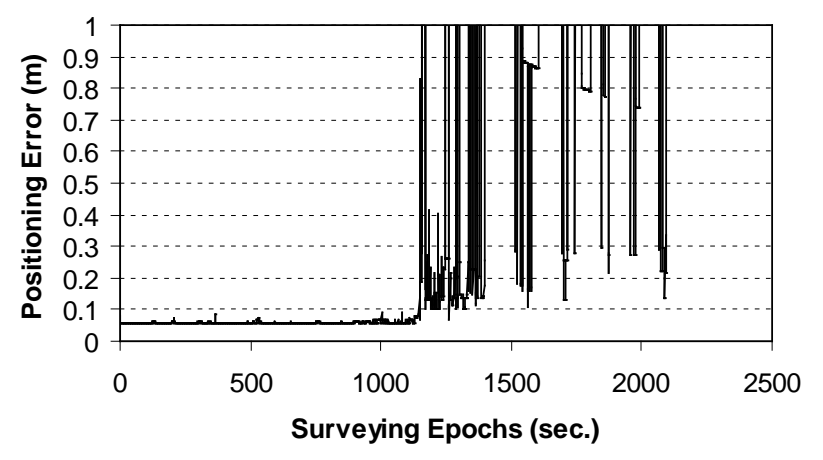

Fig. 12 Positioning Accuracy for Test Oct. 10

Three main conclusions can be drawn from the Oct.10 and 23 tests, namely:

- Tracking of satellites in the GPS handheld receivers are sensitive to dynamics, and in the kinematic mode frequent losses of phase-lock is expected, thus resulting in a decreased performance of the system.

- The five seconds of occupation of each point for determination of its coordinates is not sufficient for initialization of phase ambiguities when re-initialization is needed as a result of a change in the number of satellites tracked.

- For the successful surveying parts of the missions, the positioning accuracy achieved was slightly less than that in the static mode.

Based on results drawn from the tests of Oct.10 and Oct.23 two guidelines were set for system operation, which are:

- The occupation time per point should be increased (e.g. 30 seconds), so that no dynamics would be experienced during position determination, and to give sufficient time for re-initializing the ambiguities on the fly. This is somehow analogous to the semi-kinematic surveying methodology. The selected occupation time is however very comparable to point occupation in positioning using advanced terrestrial techniques, such as the total stations (as the time needed for sighting, focusing and labeling of target points), and slightly more than in positioning by an RTK approach using the expensive geodetic-grade GPS receivers.

- Moving between points should be carried out with low dynamics, i.e. with low velocity and acceleration, and avoiding a high level of vibrations.

These guidelines were implemented in the tests carried out on Oct.30 and Nov.6, 2002. Positioning errors in these tests are given in Figures 13 and 14, respectively, while Figures 15 and 16 show the number of satellites observed during testing. For both tests, positioning errors were generally below $6 \mathrm{~cm}$. The limited number of spikes seen in positioning errors are attributed to the decrease in the number of satellites observed to below four satellites. The conclusion given above regarding the variable behavior of the receiver in tracking of satellites in the static mode versus the kinematic one is clearly seen in Figure 16, where the number of tracked satellites for the first 600 epochs, was almost steady (7 satellites) while it started to vary when the receiver went into the kinematic mode as the observer started to move. 


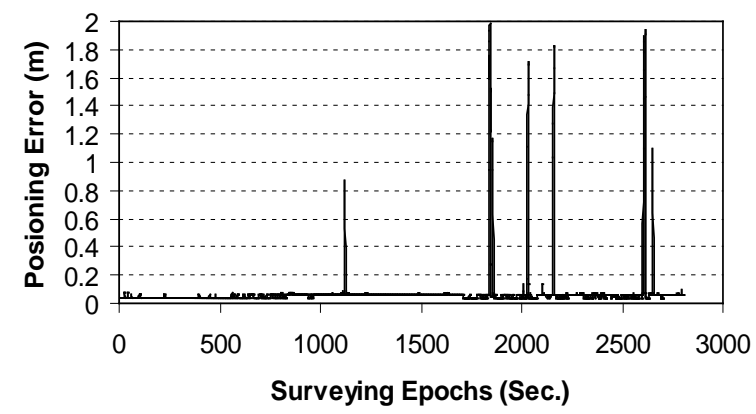

Fig. 13 Positioning accuracy for Oct.30 test

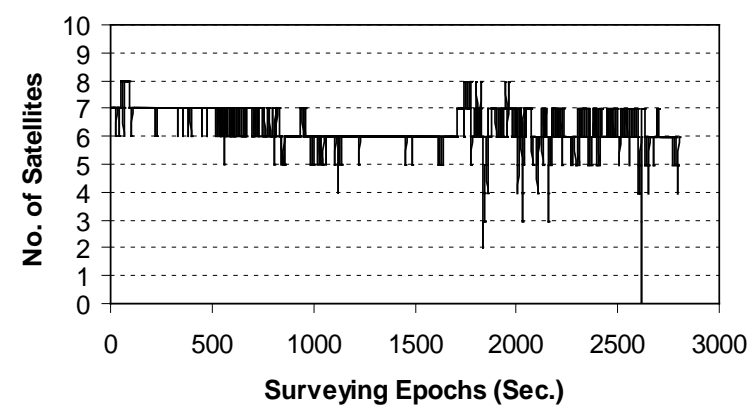

Fig. 15 No. of observed satellites in Oct.30 test

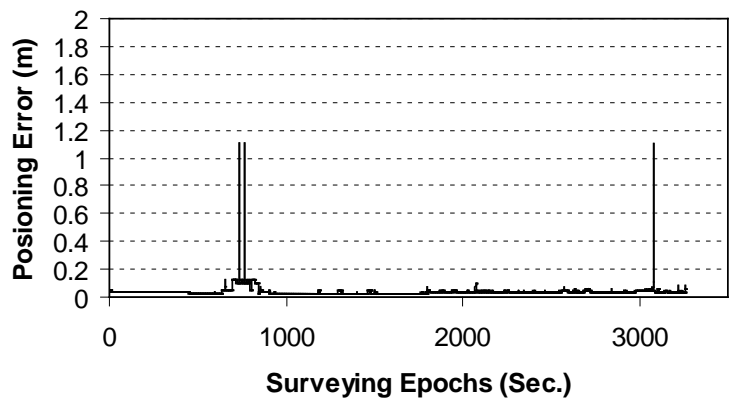

Fig. 14 Positioning accuracy for Nov.6 test

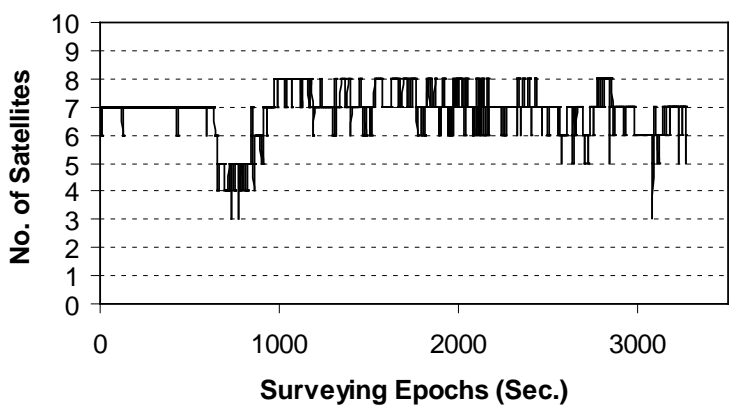

Fig. 16 No. of observed satellites in Nov.6 test

Table 2 gives a summary of the positioning accuracy results for the four given independent kinematic tests carried out. The results are given excluding the epochs of wrong position estimation due to the reasons given above. The first part of the table (columns 2 to 6) gives the average values of the standard deviations for the $\mathrm{x}, \mathrm{y}, \mathrm{z}$, and the total position estimation. The second part (columns 7 to 10) shows the maximum standard deviations estimated during processing. As can be seen, the total position standard deviation was generally less than $8 \mathrm{~cm}$. This means that, for a probability of $95 \%$, the proposed system accuracy in positioning is less than $16 \mathrm{~cm}$. The maximum error reached throughout any of the four tests was approximately $14 \mathrm{~cm}$, and with $95 \%$ probability, positioning errors are $30 \mathrm{~cm}$ at the most. This clearly reveals the success of the proposed system for position computations and generation of base maps for a wide range of GIS applications, while providing a user friendly and cheap measuring tool.

Table 2. Summary of kinematic testing results

\begin{tabular}{c|cccc|cccc}
\hline \hline & \multicolumn{4}{|c|}{ Average St. Dev.(m) } & \multicolumn{4}{c}{ Maximum St. Dev. (m) } \\
\cline { 2 - 9 } Tests & $\sigma_{\mathrm{x}}$ & $\sigma_{\mathrm{y}}$ & $\sigma_{\mathrm{z}}$ & $\sigma_{\mathrm{P}}$ & $\sigma_{\mathrm{x}}$ & $\sigma_{\mathrm{y}}$ & $\sigma_{\mathrm{z}}$ & $\sigma_{\mathrm{P}}$ \\
\hline Oct. 10 & 0.019 & 0.044 & 0.031 & 0.057 & 0.026 & 0.07 & 0.071 & 0.093 \\
\hline Oct. 23 & 0.023 & 0.056 & 0.051 & 0.080 & 0.035 & 0.105 & 0.104 & 0.128 \\
\hline Oct. 30 & 0.015 & 0.036 & 0.039 & 0.055 & 0.043 & 0.107 & 0.081 & 0.141 \\
\hline Nov. 6 & 0.016 & 0.027 & 0.024 & 0.039 & 0.056 & 0.094 & 0.082 & 0.129 \\
\hline
\end{tabular}

\section{CONCLUSIONS}

The decimeter positioning accuracy for GIS mapping can be achieved with a system that registers point information and online downloads carrier-phase data of GPS handheld receivers, and processes the data in a differential post-mission mode. The system uses only single frequency measurements, which have relatively high noise. Thus, to reach the target accuracy and aid phase ambiguity resolution, the spatially correlated errors should be minimized. This can be done by using precise orbits, choosing or establishing the reference station close to the work area, modeling of the atmospheric errors, and using archived corrections from real-time networks in a post- 
mission processing. In addition, during the process of phase ambiguity resolution, both the volume of the ambiguity search space and values of the ambiguity covariance matrix have to be greater than when using traditional phase data.

Several tests in the static and kinematic modes were carried out to test the proposed system performance. The system gave very promising results. The positioning accuracy achieved was on the decimeter level due to that handheld receivers do not have as good a capability of maintaining lock to satellites while in motion as that of the geodetic-grade receivers, and phase measurements have relatively high noise. Also, sufficient occupation time per point (approximately 30 seconds) is needed for initialization of phase ambiguities and determination of point coordinates. Future work will include studying of the system error behavior, particularly random errors such as measurement noise, multipath, and antenna phase-centre variation.

Test results showed that for a probability of $95 \%$, the positioning accuracy of the proposed system is less than $16 \mathrm{~cm}$. This reveals the success of the proposed system for position computations and generation of GIS maps. With a system cost of about $1 / 10$ of the cost of the widely used geodetic-grade GPS receivers, the results clearly prove that the system represents an efficient and economical alternative for a wide range of GIS applications.

\section{ACKNOWLEDGEMENT}

This research was financially supported by the Research Council at the UAE University under contract no. 11-7-11/02.

\section{References}

1. Campbell, C., 2001. Map Use \& Analysis. $4^{\text {th }}$ edition. McGraw-Hill Higher Education, NY.

2. Cannon, M.E., G. Lachapelle, P. Alves, L.P. Fortes, and B. Townsend, 2001. GPS RTK Positioning Using a Regional Reference Network: Theory and Results. Proceedings of GNSS 2001, The $5^{\text {th }}$ GNSS International Symposium- Satellite Navigation: Objectives and Strategies, Seville, Spain, 8-11 May, 2001

3. Enge, P., R. Fan, and A. Tiwari, 2000. GPS Reference Network New Role Providing Continuity and Coverage. GPS World, Vol. 11, No. 1.

4. Euler H., J., C.R. Keenan, B.E. Zebhauser, and G. Wübbena, 2001. Study of a Simplified Approach in Utilizing Information from Permanent Reference Station Arrays. Proceedings of ION GPS-2001, Salt Lake City, 11-14 September, 2001.

5. Fotopoulos, G., 2000. Parameterization of DGPS Carrier Phase Errors Over a Regional Network of Reference Stations. Report No. 20142. Department. of Geomatics Engineering, The University of Calgary.

6. Hill, C. J, and T. Moore, 2000. GRINGO: Recording RINEX Data from Handheld GPS Receivers. Proceedings GNSS2000, Edinburgh,1-4 May, 2000.

7. Hill, C. J, T. Moore, and M. Dumville, 2001. Carrier Phase Surveying with Garmin Handheld GPS Receivers. The Survey Review, Vol. 36, No. 280.

8. Hofmann-Wellenhof, B. H. Lichtenegger and J. Collins, 2001. Global Positioning System: Theory and Practice. $5^{\text {th }}$ edition. Springer-Verlag, Vienna.

9. Jansen, A., B. Townsend, and E. Cannon, 2002. The Use of Numerical Weather Predictions for Network RTK. Proceedings of the European Navigation Conference, the $6^{\text {th }}$ international GNSS symposium, GNSS-2002, Copenhagen, 2730 May, 2002.

10. Raquet J. and G. Lachapelle, 2001. RTK Positioning with Multiple Reference Stations. GPS World, Vol. 12, No. 4. 
11. Schuler, T. and G. Hein, 2002. Tropospheric Correction Services for GNSS UsersConcepts, Status and Future Prospects. Proceedings of the European Navigation Conference, the $6^{\text {th }}$ international GNSS symposium, GNSS-2002, Copenhagen, 2730 May, 2002.

12. Steede, K.T., 2000. Integrating GIS and the Global Positioning System. $1^{\text {st }}$ edition. ESRI Press. NY.

13. Teunissen, P. and D. Odijik, 1997. Ambiguity Dilution of Precision: Definition, Properties and Applications. Proceedings ION GPS-97, Kansas City, Missouri, September 1997.

14. Teunissen, P., P. G. de Jonge, and C. J. M. Tiberius, 1997. The Least-Squares Ambiguity Decorrelation Adjustment: Its Performance on Short GPS Baselines and Short Observation Spans. Journal of Geodesy, Vol. 71, No. 10. 